\title{
Alterations of hair cortisol and dehydroepiandrosterone in mother-infant-dyads with maternal childhood maltreatment
}

K. Schury ${ }^{1,6}$, A. M. Koenig ${ }^{1}$, D. Isele ${ }^{2}$, A. L. Hulbert ${ }^{1,3}$, S. Krause ${ }^{3}$, M. Umlauft ${ }^{4}$, S. Kolassa ${ }^{5}$, U. Ziegenhain ${ }^{6}$, A. Karabatsiakis ${ }^{1}$, F. Reister ${ }^{7}$, H. Guendel ${ }^{3}$, J. M. Fegert ${ }^{6}$ and I.-T. Kolassa ${ }^{1 *}$ (D)

\begin{abstract}
Background: Child maltreatment (CM) has severe effects on psychological and physical health. The hypothalamicpituitary-adrenal (HPA) axis, the major stress system of the body, is dysregulated after CM. The analysis of cortisol and dehydroepiandrosterone (DHEA) in scalp hair presents a new and promising methodological approach to assess chronic HPA axis activity. This study investigated the effects of CM on HPA axis activity in the last trimester of pregnancy by measuring the two important signaling molecules, cortisol and DHEA in hair, shortly after parturition. In addition, we explored potential effects of maternal CM on her offspring's endocrine milieu during pregnancy by measuring cortisol and DHEA in newborns' hair.

Methods: CM was assessed with the Childhood Trauma Questionnaire (CTQ). Cortisol and DHEA were measured in hair samples of 94 mothers and 30 newborns, collected within six days after delivery. Associations of maternal CM on her own and her newborn's cortisol as well as DHEA concentrations in hair were analyzed with heteroscedastic regression models.

Results: Higher CM was associated with significantly higher DHEA levels, but not cortisol concentrations in maternal hair. Moreover, maternal CM was positively, but only as a non-significant trend, associated with higher DHEA levels in the newborns' hair.

Conclusions: Results suggest that the steroid milieu of the mother, at least on the level of DHEA, is altered after $\mathrm{CM}$, possibly leading to non-genomic transgenerational effects on the developing fetus in utero. Indeed, we observed on an explorative level first hints that the endocrine milieu for the developing child might be altered in CM mothers. These results need extension and replication in future studies. The measurement of hair steroids in mothers and their newborns is promising, but more research is needed to better understand the effects of a maternal history of $\mathrm{CM}$ on the developing fetus.
\end{abstract}

Keywords: Cortisol, DHEA, Hair, Childhood maltreatment, Pregnancy, Transgenerational

\footnotetext{
* Correspondence: Iris.Kolassa@uni-ulm.de

${ }^{1}$ Clinical and Biological Psychology, Institute of Psychology and Education,

Ulm University, Albert-Einstein-Allee 47, 89081 Ulm, Germany

Full list of author information is available at the end of the article
} 


\section{Background}

Child maltreatment $(\mathrm{CM})$ can have detrimental psychological and biological consequences for the affected individuals and may even affect the next generation via behavioral (e.g., parenting) but probably also via genomic and non-genomic biological pathways. An elevated risk for diverse health problems in adulthood has been described in individuals with a history of $\mathrm{CM}$; for example, psychopathology, cardiovascular disease, cancer, or even premature mortality [1-3]. One possible underlying mechanism between $\mathrm{CM}$ and later physical health problems may be the dysregulation of the hypothalamic-pituitary-adrenal (HPA) axis, the body's major stress system. Functional HPA axis activity is essential for the regulation of metabolic processes in order to maintain physiological homeostasis, but its functioning can be dysregulated upon chronic stress such as CM, in particular when it occurs during sensitive developmental periods.

A stress-induced activation of the HPA axis leads to the release of corticotropin-releasing hormone (CRH) from the hypothalamus, which causes the secretion of adrenocorticotropic hormone (ACTH), and in turn results in the secretion of glucocorticoids (mainly cortisol) from the adrenal cortex. Besides cortisol, the steroid hormone dehydroepiandrosterone (DHEA) and its sulfate ester DHEA-S are released upon stress. There is growing interest in the investigation of both cortisol and DHEA, as their interplay impacts many physiological systems via genomic and non-genomic mechanisms, but they seem to have opposing biological, neurological and immune-related functions (for a review see [4]). Hence, taking both hormones into account may be a more sensitive index for the regulation of HPA axis activity [4].

A stress-induced increase of cortisol is crucial in response to environmental stressors as it results in the provision of energy by increasing the release of glucose and inhibition of non-essential functions (e.g., reproduction, growth; [4]). However, persistently altered levels of cortisol can have health-threatening effects (for more details see $[5,6]$ ).

DHEA can antagonize some of the effects of cortisol [7]. Indeed, neuroprotective, antioxidant, anti-inflammatory, and immune-modulatory effects have been described for DHEA (for details on metabolic pathways and physiological function of DHEA see e.g. [8-11]). It is related to a broad range of physiological processes, as DHEA is a precursor of many other steroid hormones (e.g., testosterone, estradiol; [8-10, 12]). Both cortisol and DHEA have been suggested as biomarkers for the regulation of HPA axis activity and related to psychiatric diseases such as depression, anxiety, PTSD, dementia, eating disorder, and externalizing problems during childhood, adolescence and even adulthood (for reviews see $[4,8]$ ).

$\mathrm{CM}$ as a chronic and traumatic stressor can lead to a persistent dysregulation of the HPA axis activity, resulting in altered cortisol secretion in affected individuals [13] and their children [14]. However, findings on longterm effects of $\mathrm{CM}$ on cortisol measured in blood, urine and saliva are mixed (e.g., [15-18]). By measuring cortisol in hair as a reliable measure of chronic HPA axis activity [19-21], several studies found an association of $\mathrm{CM}$ with lower levels of cortisol: an association of $\mathrm{CM}$ and decreased hair cortisol levels, independent of current major depression diagnosis was reported in a study with depressed patients (27 women and 16 men; mean age 41.7) and healthy age- and sex-matched controls [22]. Another study with 55 healthy college students (18 to 24 years of age) also reported a negative association of adverse childhood experiences and hair cortisol [23].

In contrast to cortisol, only few studies considered the role of DHEA in the aftermath of $\mathrm{CM}$ so far. These studies investigated CM in PTSD patients with mixed results: whereas one study observed an association of $\mathrm{CM}$ with elevated concentrations of DHEA in blood plasma of PTSD patients [24], another study investigating a sample of adult smokers with and without PTSD did not find a significant effect of CM on DHEA concentrations in blood serum [18]. In the aftermath of sexual trauma, PTSD patients showed significantly reduced DHEA levels compared to age-matched controls in two studies measuring steroids in saliva [25] and blood [26]. As all reported studies investigated DHEA in blood, the assessment of DHEA in hair as a cumulative measure over time might clarify the existing inconsistent findings.

In addition, CM may also impact the affected individual's offspring: children of mothers with a history of CM showed higher salivary cortisol concentrations compared to children of control mothers [14]. An effect of maternal traumatic experiences in adulthood on the offspring's HPA axis regulation was confirmed in infants of mothers living with PTSD, with lower cortisol concentrations in saliva [27] and blood serum [28] of the offspring.

Parental CM may affect the offspring's health and development by the dynamic interplay between environmental (e.g. parenting behavior, health behavior like smoking), and biological mechanisms (including genetic, epigenetic and non-genomic imprinting via the endocrine milieu during pregnancy). The offspring's biological constitution may be influenced during the prenatal period already, which is one of the most sensitive periods for development [29]. On the endocrine level, a dysfunctional activity of the maternal HPA axis (e.g., due to a history of $\mathrm{CM}$ ) may influence the developing HPA axis and endocrine milieu of the fetus during gestation with persisting 
effects on the offspring's HPA axis regulation and health. Indeed, during pregnancy maternal, fetal, and placental endocrine systems strongly interact in maintaining intrauterine homeostasis, ensuring maturation of vital organs in the developing fetus and the timing of parturition [30]. Whereas cortisol is essential for fetal development, as it stimulates the differentiation and maturation of vital organ systems (in particular the fetal lungs) before birth (for reviews see [30-33]), DHEA (and its sulfated form DHEAS) is critical for the physical and neural development, as it is the main source for estrogen synthesis in the placenta, which plays a pivotal role in the timing of parturition and thus for the health and survival of the newborn [30, 32]. Furthermore, DHEA is essential for brain development and is involved in neurite growth, neurogenesis, neuronal survival, and apoptosis (for reviews see $[8,9])$.

Although steroidogenesis in maternal, placental and fetal compartments is interdependent (for a review see [34]), the fetal HPA axis represents a separate biological system that is worth studying, as cortisol and DHEA, whether of fetal or maternal origin, are likely to influence development, and potentially have long-term effects on HPA function.

The analysis of cortisol and DHEA in scalp hair presents a new and promising methodological approach to measure these endocrine steroids in a non-invasive manner. Contrary to measuring acute circulating levels of cortisol and DHEA in body fluids such as blood, urine, or saliva, the assessment of cortisol and DHEA concentrations in hair allows for the retrospective analysis of the total exposure to these steroids over time [19-21]. Furthermore, the analysis of steroids in hair overcomes some of the methodological limitations when using body fluids [21] such as circadian fluctuations throughout the day.

The aim of this study was to investigate the influence of CM on the regulation of the HPA axis during pregnancy by measuring cortisol and DHEA in hair samples of postpartum women shortly after parturition. The analyzed $3 \mathrm{~cm}$ hair segment of these mothers was assumed to reflect the last trimester of pregnancy. Following previous findings on CM-related reductions in hair cortisol levels $[22,23]$, we expected a negative association of the severity of maternal CM with cortisol concentrations in the last trimester of pregnancy measured in hair of postpartum mothers. Furthermore, we also explored an association of $\mathrm{CM}$ and DHEA levels in the last trimester of pregnancy measured in the hair of postpartum women. In addition, we tested a potential association of the mothers' $\mathrm{CM}$ experiences and her offspring's HPA axis in an explorative analysis. Cortisol and DHEA were measured in the newborn's hair, which were assumed to reflect fetal HPA axis activity before parturition.

\section{Methods}

\section{Study design and population}

Mothers and their newborns were recruited in the maternity ward of the University Hospital Ulm, Germany. Exclusion criteria were maternal age under 18 years of age, insufficient knowledge of the German language, severe complications during parturition, severe health problems of mother or child, as well as maternal drug consumption or psychotic disorders. Of 1460 mothers that were invited to participate, a total of 240 gave written informed consent within six days after parturition $($ median $=2$ days; range $=[0 ; 6]$ days $)$; basic sociodemographic information was assessed and mothers were screened for CM experiences using the German version of the Childhood Trauma Questionnaire (CTQ; [35]).

To ensure sufficient statistical power to detect longterm effects of maltreatment experiences, we oversampled for higher CTQ sum scores: Based on the distribution of CTQ sum scores obtained in a pilot study with $N=185$ postpartum women (unpublished data), the CTQ scale was divided in $25 \%$ quantiles, resulting in the following value ranges: $25-28$ (25\%), 29-32 (50\%), 33-39 (75\%) and 40-125 (100\% quantile). We aimed at the same number of women in each value range at a three-month follow-up (cf. below and Additional files 1, 2 and 3). As expected, the distribution of CTQ sum scores was skewed to the right. Thus, we recruited all women with CTQ sum scores $\geq 40$. For every woman with a sum score $\geq 40$ who participated at the three-month follow-up, we consecutively included one women in the other value ranges. To compensate for drop-outs between $t_{0}$ and follow-up, value ranges were filled up each time when a woman dropped out at follow-up, so that slightly more women were recruited at $t_{0}$ than in an ideal situation without drop-outs. This led to the inclusion of 112 mothers at $t_{0}$ (28 with CTQ sum score $\geq 40 ; 26$ in the range 33-39, 21 with scores between 29 and 32 range and 37 with scores $\leq 29$ ), of which hair samples could be obtained from 106 mothers and 49 newborns. Samples of one mother and 19 infants had to be excluded as the hair sample weight was below the minimum of $5 \mathrm{mg}$. Mothers with any intake of medication during pregnancy with known effects on HPA axis activity (e.g. glucocorticoids) were excluded. This led to the additional exclusion of another eleven mothers (no further hair samples of newborns had to be excluded). The final sample consisted of $N=94$ mothers and $N=30$ newborns. Study procedures and drop-outs are described in Additional file 1. Participants received $10 €$ for study participation at $t_{0}$. All procedures were approved by the ethics committee of Ulm University.

At the time of recruitment, mean age of the 94 women was 32.45 years (range $=[21 ; 44], S D=5.44$ years). Gestation lasted between 37 and 42 weeks $($ mean $=39.70$, 
$S D=1.37)$. Further sociodemographic and medical data is reported in Table 1.

Mothers and newborns who were excluded due to low weight of hair sample or medication during pregnancy did not differ from mothers and newborns included with respect to $\mathrm{CM}$ experiences, maternal age, weeks of gestation, infants' birth weight, marital status, education level, or maternal perceived stress in the last month of pregnancy (all $p>.05$ ).

In a subsample of $N=42$ mothers, more detailed information on adverse childhood experiences was

Table 1 Descriptive data for the 94 mothers and 30 newborns

\begin{tabular}{|c|c|}
\hline Variable & $\begin{array}{c}M \pm S D \\
N(\%)\end{array}$ \\
\hline \multicolumn{2}{|l|}{ Maternal data $(N=94)$} \\
\hline \multicolumn{2}{|l|}{ Hair steroids } \\
\hline Cortisol in pg/mg & $18.89 \pm 74.51$ \\
\hline DHEA in $\mathrm{pg} / \mathrm{mg}$ & $4.33 \pm 11.00$ \\
\hline \multicolumn{2}{|l|}{ Medical and demographic data: } \\
\hline Age in years & $32.45 \pm 5.44$ \\
\hline Married & $72(76.60 \%)$ \\
\hline German origin & $83(88.3 \%)$ \\
\hline Higher school education (> 11 years of school) & $55(58.51 \%)$ \\
\hline Hair treatment & $34(36.17 \%)$ \\
\hline - Colored hair & $18(19.15 \%)$ \\
\hline - Permanent wave & $3(3.19 \%)$ \\
\hline - Dyeing & $13(13.83 \%)$ \\
\hline$B M I^{\mathrm{a}}$ & $24.54 \pm 4.79$ \\
\hline Currently smoking & $9(9.57 \%)$ \\
\hline Time between parturition and hair collection (days) & $2.20 \pm 1.16$ \\
\hline \multicolumn{2}{|l|}{ Psychological data } \\
\hline Child maltreatment (CTQ sum score) & $36.29 \pm 12.96$ \\
\hline Perceived Stress (PSS4) & $4.59 \pm 3.05$ \\
\hline Lifetime psychopathology & $27(28.72 \%)$ \\
\hline - Major Depression & 17 \\
\hline - Anxiety & 7 \\
\hline - Other & 12 \\
\hline \multicolumn{2}{|l|}{ Newborn data $(N=30)$} \\
\hline \multicolumn{2}{|l|}{ Hair steroids } \\
\hline Cortisol in pg/mg & $208.00 \pm 122.02$ \\
\hline DHEA in $\mathrm{pg} / \mathrm{mg}$ & $3.31 \pm 1.70$ \\
\hline \multicolumn{2}{|l|}{ Medical data } \\
\hline Gestational age at birth ${ }^{b}$ & $39.77 \pm 1.51$ \\
\hline Birth weight in gramb & $3328 \pm 482.35$ \\
\hline
\end{tabular}

$M=$ mean; $S D=$ standard deviation; $N \%$ = relative frequency in valid percent; $B M I=$ body mass index; $C T Q=$ Childhood Trauma Questionnaire;

PSS4 = Perceived Stress Scale, four-item version

${ }^{\mathrm{a}}$ Missing data for $\mathrm{BMI}$ in 35 cases

${ }^{\mathrm{b}}$ Missing data in 8 newborns assessed with the German version of the Maltreatment and Abuse Chronology of Exposure (MACE; [36, 37]) three months after parturition. For the interested reader, we report recruitment and data assessment, study procedure and results regarding the association of MACE scores and HPA measurements in hair of these $N=42$ mothers and their $N=15$ newborns in Additional files 1, 2, and 3 respectively.

\section{Psychological measures Maltreatment load}

$\mathrm{CM}$ was measured with the German version of the short form of the Childhood Trauma Questionnaire (CTQ; [35]). Experiences of emotional abuse, physical abuse, sexual abuse, emotional neglect, and physical neglect are assessed with five items each and rated on a five-point Likert scale. Ratings of the 25 items were summed up, with higher values indicating higher maltreatment load.

\section{Covariates}

The following demographic, medical and psychological data were assessed as potential confounding variables on the effect of childhood maltreatment on hair steroids: maternal age, body mass index (BMI), smoking status, hair treatment (i.e., coloring, permanent wave or dyeing) in the past three months, perceived stress during the last month (PSS4; [38]), as well as gestational age and birth weight. The prevalence of maternal lifetime psychiatric diagnoses was assessed via self-report with the question whether one had ever been diagnosed with a psychiatric disorder during one's lifetime.

\section{Collection of hair and analysis of cortisol and DHEA using HPLC-coupled mass spectrometry}

Hair strands were cut from the posterior vertex region of the mothers within six days after parturition as close to the scalp as possible. In order to gain enough material for analyses ( $>5 \mathrm{mg})$, two to three hair strands $(\sim 3 \mathrm{~mm}$ diameter) were collected following recommendations provided by the Society of Hair Testing [39]. In the infants, two to three hair strands were obtained following the same protocol. However, as hair in newborns is sparse, hair strands were cut at locations with most hair, most often at the hairline beneath the ear. Hair samples were then wrapped in aluminum foil and stored at $-20 \mathrm{C}^{\circ}$ until shipping to the laboratory of Prof. Dr. Kirschbaum, Dresden, Germany, for analysis. Maternal hair strands of $3 \mathrm{~cm}$ adjacent to the scalp were analyzed, reflecting the last trimester of pregnancy, given an average hair growth of $1 \mathrm{~cm}$ per month [40]. Since studies on neonatal hair growth suggested that the metabolic activity of prenatal development in the third trimester of pregnancy is reflected in neonatal hair fibers present at birth [41], the whole hair strand was analyzed in newborns. Maternal 
hair samples were analyzed in different batches, newborns' hair in one batch.

Washing and extraction of cortisol and DHEA were performed according to the protocol of Gao and colleagues [20]. In short, every hair segment was washed twice in isopropanol and then dried for at least $12 \mathrm{~h}$. Using the same hair segment, cortisol and DHEA were extracted from non-pulverized hair in methanol at room temperature overnight. After centrifugation at 10,000 rpm for two minutes, the supernatant was used and the methanol was evaporated at $65{ }^{\circ} \mathrm{C}$ under a constant nitrogen stream. Afterwards, the dried samples were resuspended in $150 \mu \mathrm{l}$ double-distilled water. A Shimadzu HPLCtandem mass spectrometer (Shimadzu, Canby, OR), which was coupled to an ABSciex API 5000 Turbo ion-spray triple quadruple tandem mass spectrometer (AB Sciex, Foster City, CA) was used for the analysis.

\section{Statistical analyses}

All statistical analyses were conducted with R 3.2.5 [42] using a 5\% level of significance. For descriptive purposes, information on mean values and standard deviations $(S D)$, as well as data shown in graphs are presented in original units (pg hormone/mg hair). For correlation analyses, the non-parametric Kendall's Tau is reported as distribution of hair data is skewed.

Typical restrictions of standard linear models are normally distributed error terms and equal variances across groups. Since these assumptions were violated, standard methods would have been not valid. If the assumption of homogeneity of variance is violated, heteroscedastic regression analysis are known to stabilize the variance of regression estimates [43] and thus, were used to draw meaningful conclusions from the present data. In short, Ordinary Least Squares (OLS) models were estimated on $10^{4}$ bootstrap samples and the means of the generated $b^{\prime}$ coefficients and standard errors, as well as $t$-statistic and $F$-statistic respectively, and $p$ values are reported. For bootstrapping the residuals we used the wild bootstrap technique from the fANCOVA package [44]. For more details see Liu [45] and Rana et al. [43]. Covariates (see above) for the regression models were chosen based on Akaike's Information Criterion (AIC; [46]). No significant differences between the batches were observed in maternal cortisol data ( $W=895.5, p=.22)$. However, maternal DHEA concentrations significantly differed between the batches (Wilcoxon rank sum tests: $W=668.5$, $p=.003$ ). Thus, a dummy variable coding for the batch effect was included as a covariate in regression analyses with maternal DHEA data.

A simple plot identified one particularly high cortisol score of $706.76 \mathrm{pg} / \mathrm{mg}$ as well as one particularly high DHEA score of $90.38 \mathrm{pg} / \mathrm{mg}$ in maternal hair data. In a chi-squared test for outliers ([47]; using R Package 'outliers' by Komsta; [48]) these scores significantly diverged from the distribution of all other cortisol or DHEA data (cortisol: $x^{2}=85.24, p<.001$; DHEA: $\left.X^{2}=61.14, p<.001\right)$ and were thus excluded in correlation and regression analyses with maternal hair steroids were to prevent these scores from hampering the statistical analyses.

\section{Results}

A total of 21 (22.34\%) mothers reported at least low to moderate levels of emotional abuse, 11 (11.70\%) physical abuse, 15 (15.96\%) sexual abuse, 44 (46.81\%) emotional neglect, and $11(11.70 \%)$ physical neglect in childhood according to cut-off scores described for the CTQ by Bernstein et al. [49]. CTQ sum scores ranged from 25 to 103 with a mean of $36.29(S D=12.96)$. Descriptive data on cortisol and DHEA concentrations in hair of women during pregnancy and their newborns, maternal exposure to childhood maltreatment, as well as medical and psychological data are reported in Table 1.

Cortisol and DHEA levels were not significantly correlated with each other in women (Kendall's $\tau=0.04$, $p=.53$ ) or newborns (Kendall's $\tau=0.13, p=.33$ ). Hair DHEA concentrations in hair of mothers were significantly correlated with DHEA levels in newborn's hair (Kendall's $\tau=0.30, p=.003$ ). Maternal and newborns' hair cortisol concentrations were not significantly correlated (Kendall's $\tau=0.12, p=.25$ ).

\section{Association of $\mathrm{CM}$ with cortisol and DHEA levels in the last trimester of pregnancy measured in maternal hair} A regression model with $\mathrm{CM}$ as an independent variable and hair cortisol as a dependent variable without further covariates minimized the AIC. No significant association of CM on hair cortisol was obtained (see Table 2).

We observed a positive association of CM (CTQ sum score) and hair DHEA concentrations ( $b=0.12 ; p=.01)$ controlling for batch effects in which the hair samples were analyzed $(b=3.49 ; p=.01)$. All variables explained $20 \%$ of the variance in DHEA $(F(2,90)=11.66$, $p<.001)$. This association remained significant after controlling for maternal age and perceived stress as covariates (chosen by the AIC scores), as well as the hair batch in heteroscedastic regression (see Table 2). The full model explained $25 \%$ of the variation in hair DHEA $(F(4,89)=6.91$, $p<.001)$. Results are illustrated in Fig. 1 .

\section{Association of maternal CM on cortisol and DHEA levels measured in the newborn's hair}

Maternal CM was not significantly associated with prenatal cortisol levels in newborns' hair $(b=2.42, p=.18)$. Including maternal age as covariate minimized the AIC. Similarly, no significant effect of the CTQ sum score on 
Table 2 Summary of heteroscedastic regression analyses for variables predicting cortisol and DHEA in hair samples of mothers and their newborns

\begin{tabular}{lcccc}
\hline Maternal hair data & & & & \\
Cortisol concentrations $\left(N=93^{\mathrm{a}}\right)$ & $b$ & $S E$ & $t(91)$ & $p$ \\
Childhood maltreatment (CTQ sum score) & 0.12 & 0.15 & 0.72 & .471 \\
$\qquad R^{2}=.02, F(1,91)=1.08, p=.302$ & & & \\
DHEA concentrations $\left(N=93^{+}\right)$ & $b$ & $S E$ & $t(91)$ & $p$ \\
Childhood maltreatment (CTQ sum score) & 0.28 & 0.08 & 3.25 & $.002^{* *}$ \\
Age & 0.51 & 0.19 & 2.56 & $.012^{*}$ \\
Perceived stress (PSS4) & -0.58 & 0.37 & -1.41 & .16 \\
Hair batch & 2.48 & 2.10 & 1.29 & .20 \\
$R^{2}=.25, F(4,89)=6.91, p<.001^{* *}$ & & &
\end{tabular}

Newborns' hair data

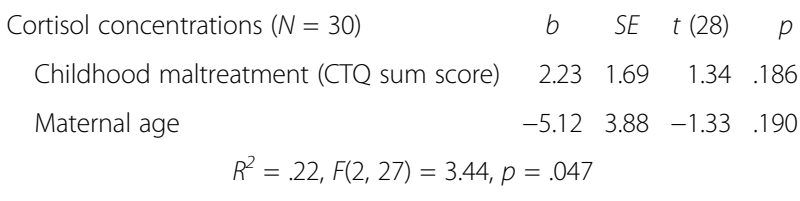

DHEA concentrations $(N=30) \quad b \quad$ SE $t(28) \quad p$

$\begin{array}{lllll}\text { Childhood maltreatment (CTQ sum score) } & 0.04 & 0.02 & 1.89 & .070^{\#}\end{array}$ $R^{2}=.17, F(1,28)=4.79, p=.037^{*}$

${ }^{\#}<.10,{ }^{*}<.05,{ }^{* *}<.01,{ }^{* * *}<.001$

ane outlier in DHEA, and cortisol respectively, was excluded from regression analysis

$C T Q=$ Childhood Trauma Questionnaire; PSS4 = four-item version of the Perceived Stress Scale

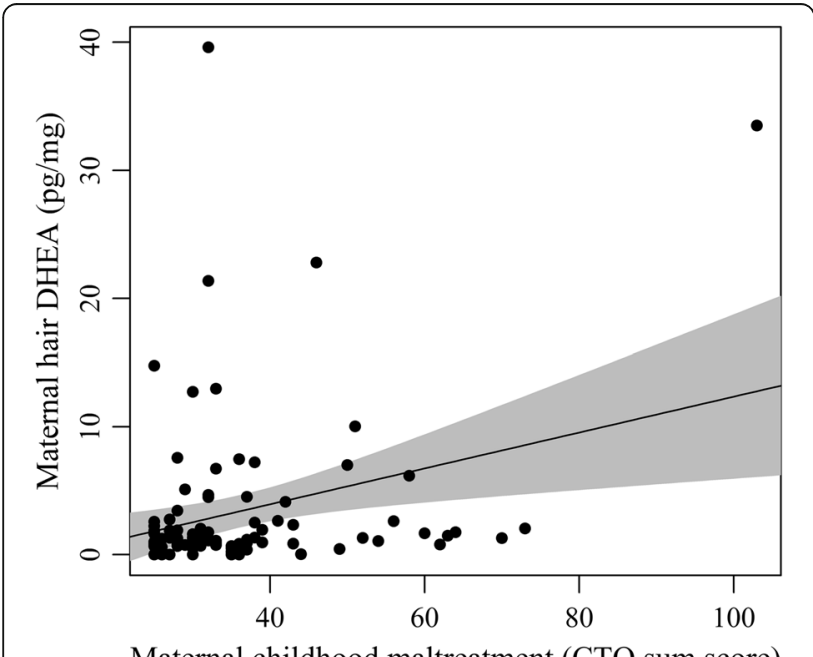

Maternal childhood maltreatment (CTQ sum score)

Fig. 1 Regression line of maternal childhood maltreatment and DHEA concentrations in maternal hair, reflecting the third trimester of pregnancy. The grey area depicts the pointwise 95\% confidence area prenatal cortisol in hair of newborns was found when controlling for maternal age (see Table 2).

A main effect model of maternal CM (CTQ sum score) as an independent variable for prenatal DHEA levels measured in hair of the newborns as a dependent variable without further covariates was chosen based on the AIC for the analyses. Maternal CM was positively associated with prenatal DHEA levels in newborns, but as a non-significant trend $(b=.04 ; p=.07) .17 \%$ of the variance in DHEA concentrations was explained by maternal $\mathrm{CM}(F(1,28)=4.79 ; p=.04)$. Results are shown in Table 2 and illustrated in Additional file 4.

\section{Discussion}

In contrast to previous findings, hair cortisol levels were not associated with the amount of CM experienced in our sample of postpartum women. Prior studies reported a negative association between $\mathrm{CM}$ and hair cortisol [22, 23]. However, our results are in line with another study reporting no such association [50]. However, none of these studies investigated steroids during pregnancy. Differing results may be explained by the physiological and endocrine alterations in the last trimester of pregnancy, which might mask the replicated finding of lower cortisol in the aftermath of CM. In short, CRH is now also secreted by the fetal adrenal gland, the placenta and decidua, and consequently contributes to an exponential increase of maternal plasma CRH. Due to elevated CRH as well as a longer half-life of cortisol in plasma (as a result of estrogen-induced reduction of cortisol catabolism in the liver) cortisol levels increase with a peak during the third trimester of pregnancy (for more details see [33]). The one study investigating hair cortisol in relation to $\mathrm{CM}$ in pregnant women confirmed our results of no association in Caucasian pregnant women with relatively low levels of CM [51].

Our finding of elevated DHEA levels in hair is in accordance to some studies measuring DHEA in body fluids of PTSD patients [24, 52, 53]: Significant amounts of the variation of elevated DHEA were explained by childhood trauma history in women with PTSD [24]. However, other studies did not find an association of CM with DHEA, but with cortisol in blood plasma and serum of PTSD patients [18, 54]. Studies investigating DHEA in hair are lacking, thus further studies are needed to clarify effects of CM on chronic DHEA levels.

Furthermore, this study explored the influence of maternal CM on the next generation's HPA axis regulation via non-genomic effects by influencing the endocrine milieu in utero of the developing fetus. In contrast to previous findings, we did not observe an association of maternal CM and cortisol levels in the newborn's hair. However, in previous studies that related maternal CM [14] or PTSD $[27,55]$ with lower cortisol concentrations, 
cortisol was measured in saliva of the offspring at the age of six months or even adulthood. Thus, results are not comparable to our findings regarding prenatal steroid concentrations measured in newborns' hair, as prenatal HPA axis activity differs significantly from HPA axis functioning during childhood or adulthood and does not allow retrospective conclusions about the inutero environment of the developing fetus.

Regarding DHEA, higher maternal CM was positively associated with elevated DHEA concentrations in the hair of our newborn sample but only as a nonsignificant trend $(p=.07)$, explaining $17 \%$ of the variation in hair DHEA. This relationship was significant in a subsample of newborns $(N=15)$ whose mothers were interviewed in detail about a broader range of adverse childhood experiences including also witnessing physical violence towards parents or siblings, peer emotional violence, and peer physical violence (see Additional file 2). However, as the sample size of this subsample was small, this finding should be seen as hypothesis-generating for future studies, and thus no final conclusions should be drawn at this stage (see Additional files 3 and 4). The only other study investigating cumulative prenatal DHEA concentrations measured DHEA in the fingernails of newborns whose mothers had experienced stress during pregnancy and report a positive association [56]. However, they did not examine maternal lifetime stress such as adverse childhood experiences.

This results hint towards a potential effect of maternal childhood experiences on the intrauterine endocrine environment of the developing offspring in late gestation, but presumably also during the whole gestational process. Indeed, $\mathrm{CM}$ has been associated with altered HPA axis activity during pregnancy of affected mothers [51]; and a dysfunctional activity of the maternal HPA axis during gestation has been associated with alterations in the offspring's HPA axis [57, 58]. Altered levels of steroids in the mother during pregnancy might influence the fetal HPA axis directly by crossing the placental barrier, and indirectly by stimulating placental CRH production and reducing uteroplacental blood flow [59]. Thus, the developing fetus might achieve and incorporate information about its later environment via intrauterine maternal-placental-fetal communication - a phenomenon referred to as "fetal programming" [60, 61]. By programming of the fetal HPA axis, the offspring may adapt to the environment in which they will be born in anticipation of an exposure to similar environmental conditions in postnatal life [57, 62]. However, results in this study regarding a potential transgenerational effect of $\mathrm{CM}$ were only significant on a trend level or were only present in a subsample for which also information on witnessing physical violence towards parents or siblings, peer emotional violence, and peer physical violence was available. Thus, our findings need replication in larger samples but can be used for the formulation of hypotheses and calculation of effect sizes for future studies.

\section{Strengths and limitations}

One major limitation of this study is the relatively small sample of newborns with sufficient amount of hair for analysis due to the limited availability of sufficient hair for analysis. Studies on neonatal hair growth suggested that neonatal hair fibers at birth reflect the metabolic activity of prenatal development in the third trimester of pregnancy [41]. Nevertheless, length of maternal and newborn hair samples differed $(3 \mathrm{~cm}$ in mothers vs. approximately $1 \mathrm{~cm}$ in newborns) and might not represent the exact same time period. In addition, cortisol and DHEA concentrations measured in the newborns' hair may be of fetal and, partly, of maternal origin: Even though fetal exposure to maternal steroids is limited due to the actions of the placental 11ß-HSD2 enzyme, which transforms cortisol into its inactive form cortisone and thus provides a partial barrier [59, 63, 64], a small percentage of maternal glucocorticoids still passes the placenta into the fetal system (for reviews see e.g. [65]). Furthermore, methodological issues (variability associated with growth rate of hair and inconsistencies in collection of hair samples $[66,67])$ as well as incorporation of steroids in hair [68-73] are still discussed. Besides incorporation via blood during formation of hair follicles, external sources such as sweat or, in the case of newborns, amniotic fluid may also affect hair that has already emerged on the scalp $[67,71]$.

Further limitations are related to the psychological data assessment: Whereas the maternal steroids in the $3 \mathrm{~cm}$ hair segment reflected the last three months, only the perceived stress in the last month was considered as a covariate in the analyses (assessed with the PSS4, which instructs participants to refer to the past month). Furthermore, the covariate lifetime psychopathology was only assessed in self-report and not with a standardized psychiatric interview.

Strengths of this study are the assessment of steroids in hair as a cumulative measure of HPA axis activity, the consideration of two important steroids (cortisol and DHEA) and the investigation of hair samples of mothernewborn dyads extending existing literature on the CMrelated transmission of HPA axis dysregulation, which most often confined analysis of steroid hormones to the offspring generation. Furthermore, the influence of parental behavior on steroid levels in the hair of newborns can be nearly excluded in this study, since hair samples were collected within six days after parturition. Remarkably, we observed an effect of CM on HPA axis activity in a sample of well-educated healthy women with relatively low psychosocial burden (only five women were 
worried about financial problems due to the child's birth, four reported cramped living conditions [ratio of number of rooms/number of persons in the flat $\leq 0.5]$, and one woman experienced mild physical violence by her intimate partner within the last 8 weeks). However, results can thus only be generalized to a limited degree and studies on high-risk samples are needed.

\section{Implications for research and conclusion}

Results of this study revealed an association of increasing cumulative DHEA, but not cortisol levels in maternal hair with rising number of $\mathrm{CM}$ experiences. This might reflect long-term alterations in the HPA axis in response to maltreatment experiences in childhood, which extend into pregnancy and highlights the importance to investigate DHEA in addition to cortisol. With due caution, our data also provide some support for the hypothesis that CM may influence the offspring's prenatal HPA axis, as we observed a positive association of maternal $\mathrm{CM}$ and elevated DHEA concentrations in the newborns' hair, but as a nonsignificant trend.

Measuring cumulative cortisol and DHEA concentrations in mothers and their newborns shortly after parturition seems promising for the investigation of prenatal biological effects of maternal trauma history on her offspring's neuroendocrine system, without the confounding effect of parental behavior.

Further studies in larger (high-risk) samples are needed and would allow further analyses (e.g., taking ongoing life circumstances, time between CM and pregnancy, as well as the specific effects of different types of CM into account), in order to better understand the underlying biological mechanisms in the interplay between $\mathrm{CM}$ and a potentially altered HPA axis activity. Furthermore, longitudinal studies should investigate whether altered levels of DHEA in late gestation are related to altered patterns of HPA axis activity in childhood and adulthood. Pregnancy is associated with significant physiological alterations of HPA axis functioning and the biological function as well as implications of elevated DHEA in non-pregnant individuals cannot easily be transferred into the third trimester of pregnancy. Thus, long-term effects of increased DHEA levels in late gestation on health outcomes in the mothers and their offspring needs to be addressed in prospective studies and the investigation of hair DHEA levels in non-pregnant women with a history of CM seems warranted.

\section{Additional files}

Additional file 1: Study procedure and drop-outs. Figure illustrating the study procedure (measurement points) and reporting drop-out rates and reasons for drop-out. (PDF $151 \mathrm{~kb}$ )
Additional file 2: Description of the data assessment using the MACE to assess adverse childhood experiences. Additional file 2 describes the procedure for the additional data assessment (i.e., a more detailed interview about adverse childhood experiences) with a subsample of mother-infant dyads three months postpartum. (PDF $152 \mathrm{~kb}$ )

Additional file 3: Summary of heteroscedastic regression analyses for variables predicting DHEA, and cortisol in hair samples of mothers and their newborns, who participated in three-months follow-up and were interviewed with the MACE. Table with results from heteroscedastic regression analyses for the subsample of participants described in Additional file 2. (PDF $132 \mathrm{~kb}$ )

Additional file 4: Regression line of maternal childhood maltreatment assessed by CTQ (left panel) and MACE (right panel) respectively and prenatal DHEA concentrations measured in newborn's hair. The grey area shows the pointwise $95 \%$ confidence area. The figure illustrates the association of adverse childhood experiences (comparing two different instruments) and concentrations of DHEA in hair in a subsample of newborns (described in Additional file 2). (PDF $231 \mathrm{~kb}$ )

\section{Abbreviations}

ACTH: Adrenocorticotropic hormone; AlC: Akaike's Information Criterion; BMI: Body mass index; CM: Childhood maltreatment; CRH: Corticotropinreleasing hormone; CTQ: Childhood Trauma Questionnaire;

DHEA: Dehydroepiandrosterone; HPA axis: Hypothalamic-pituitary-adrenal axis; M: Mean; MACE: Maltreatment and Abuse Chronology of Exposure; OLS: Ordinary least squares; PSS4: Perceived Stress Scale, 4-item version; PTSD: Posttraumatic stress disorder; SD: Standard deviation

\section{Acknowledgements}

We thank Sarah Wilker for comments on an earlier version of the manuscript and Traudl Hiller (medical technical assistant) for support in data collection.

\section{Availability of data and materials}

The datasets generated and/or analyzed during the current study are not publicly available due to them containing information that could compromise research participant privacy/consent but are available from the corresponding author on reasonable request in anonymized form.

\section{Funding}

Katharina Schury received a scholarship from the German National Academic Foundation (Studienstiftung des deutschen Volkes). Alexandra Koenig was supported by a scholarship of the Konrad Adenauer Foundation. The work of Maria Umlauft was supported by the German Research Foundation project DFG-PA 2409/3-1. The funding bodies neither influenced the design of the study, nor collection, analysis, and interpretation of data, and did not affect writing of the manuscript. Data acquisition was funded by university resources of I.-T. Kolassa. The analysis of DHEA and cortisol in hair were funded by university resources of I.-T. Kolassa and J. M. Fegert.

\section{Authors' contributions}

KS and ITK with support of UZ, HG, AK and JMF were responsible for the conception and design of the study. Acquisition of data was conducted by $\mathrm{KS}, \mathrm{DI}, \mathrm{ALH}, \mathrm{FR}$, and SKr. KS, and MU analyzed and interpreted the data together with AMK, SKo, and ITK. KS was the major contributor in writing the manuscript with support of AMK. KS, DI, ALH, MU, AMK, SK, UZ, AK, FR, HG, JMF and ITK critically revised the manuscript for important intellectual content. All authors approved the final version of the manuscript and agreed to be accountable for all aspects of the work in ensuring that questions related to the accuracy or integrity of any part of the work are appropriately investigated and resolved.

\section{Authors' information}

Not applicable.

\section{Competing interests}

The authors declare that they have no competing interests.

Consent for publication

Not applicable. 


\section{Ethics approval and consent to participate}

All study procedures are in accordance with the Declaration of Helsinki and were approved by the local ethical committee of UIm University (reference number 341/11). All participants provided written informed consent before participation of themselves and their children.

\section{Publisher's Note}

Springer Nature remains neutral with regard to jurisdictional claims in published maps and institutional affiliations.

\section{Author details}

${ }^{1}$ Clinical and Biological Psychology, Institute of Psychology and Education, Ulm University, Albert-Einstein-Allee 47, 89081 Ulm, Germany. ${ }^{2}$ Department of Psychology, University of Konstanz and vivo international, 78457 Konstanz, Germany. ${ }^{3}$ Department of Psychosomatic Medicine and Psychotherapy, University Hospital Ulm, 89081 Ulm, Germany. ${ }^{4}$ Institute of Statistics, UIm University, 89081 Ulm, Germany. ${ }^{5}$ SAP Switzerland, 8274 Tägerwilen, Switzerland. ${ }^{6}$ Department of Child and Adolscent Psychiatry and Psychotherapy, University Hospital Ulm, 89075 Ulm, Germany. ${ }^{7}$ Department of Obstetrics and Gynecology, University Hospital UIm, 89075 Ulm, Germany.

\section{Received: 12 November 2016 Accepted: 19 May 2017}

\section{Published online: 06 June 2017}

\section{References}

1. Carr CP, Martins CMS, Stingel AM, Lemgruber VB, Juruena MF. The role of early life stress in adult psychiatric disorders: a systematic review according to childhood trauma subtypes. J. Nerv. Ment. Dis. 2013;201:1007-20. doi:10.1097/NMD.0000000000000049.

2. Brown DW, Anda RF, Tiemeier H, Felitti VJ, Edwards VJ, Croft JB, et al. Adverse childhood experiences and the risk of premature mortality. Am J Prev Med. 2009;37:389-96. doi:10.1016/j.amepre.2009.06.021.

3. Felitti VJ, Anda RF, Nordenberg D, Williamson DF, Spitz AM, Edwards V, et al. Relationship of childhood abuse and household dysfunction to many of the leading causes of death in adults. Am J Prev Med. 1998;14:245-58. doi:10.1016/S0749-3797(98)00017-8.

4. Kamin HS, Kertes DA. Cortisol and DHEA in development and psychopathology. Horm Behav. 2016;89:69-85. doi:10.1016/j.yhbeh.2016.11.018.

5. Myers B, McKlveen JM, Herman JP. Neural regulation of the stress response: the many faces of feedback. Cell Mol Neurobiol. 2012;32:683-94. doi:10.1007/s10571-012-9801-y.

6. Smith SM, Vale WW. The role of the hypothalamic-pituitary-adrenal axis in neuroendocrine responses to stress. Dialogues Clin Neurosci. 2006;8:383-95.

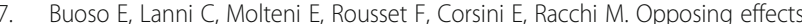
of cortisol and dehydroepiandrosterone on the expression of the receptor for activated C kinase 1: implications in immunosenescence. Exp Gerontol. 2011;46:877-83. doi:10.1016/j.exger.2011.07.007.

8. Maninger N, Wolkowitz OM, Reus VI, Epel ES, Mellon SH. Neurobiological and neuropsychiatric effects of dehydroepiandrosterone (DHEA) and DHEA sulfate (DHEAS). Front Neuroendocrinol. 2009;30:65-91. doi:10.1016/j.yfrne.2008.11.002.

9. Pluchino N, Drakopoulos P, Bianchi-Demicheli F, Wenger JM, Petignat $P$, Genazzani AR. Neurobiology of DHEA and effects on sexuality, mood and cognition. J Steroid Biochem Mol Biol. 2015;145:273-80. doi:10.1016/j.jsbmb. 2014.04.012

10. Stárka L, Dušková M, Hill M. Dehydroepiandrosterone: a neuroactive steroid. Steroid Biochem Mol Biol. 2015;145:254-60. doi:10.1016/j.jsbmb.2014.03.008.

11. Traish AM, Kang P, Saad F, Guay AT. Dehydroepiandrosterone (DHEA) - a precursor steroid or an active hormone in human physiology. J Sex Med. 2011;8:2960-82. doi:10.1111/j.1743-6109.2007.00413.x.

12. Labrie F. DHEA, important source of sex steroids in men and even more in women. Prog Brain Res. 2010;182:97-148. doi:10.1016/S0079-6123(10)82004-7.

13. van Voorhees EE. The effects of child maltreatment on the hypothalamicpituitary-adrenal axis. Trauma, Violence, \& Abuse. 2004;5:333-52. doi:10.1177/1524838004269486.

14. Brand SR, Brennan PA, Newport DJ, Smith AK, Weiss T, Stowe ZN. The impact of maternal childhood abuse on maternal and infant HPA axis function in the postpartum period. Psychoneuroendocrinology. 2010;35:686-93. doi:10.1016/j.psyneuen.2009.10.009.

15. Carpenter LL, Carvalho JP, Tyrka AR, Wier LM, Mello AF, Mello MF, et al. Decreased adrenocorticotropic hormone and cortisol responses to stress in healthy adults reporting significant childhood maltreatment. Biol Psychiatry. 2007;62:1080-7. doi:10.1016/j.biopsych.2007.05.002.

16. Harkness KL, Stewart JG, Wynne-Edwards KE. Cortisol reactivity to social stress in adolescents: role of depression severity and child maltreatment. Psychoneuroendocrinology. 2011;36:173-81. doi:10.1016/j.psyneuen.2010.07.006.

17. Doom JR, Cicchetti D, Rogosch FA. Longitudinal patterns of cortisol regulation differ in maltreated and nonmaltreated children. Journal of the American Academy of Child \& Adolescent Psychiatry. 2014;53:1206-15. doi:10.1016/j.jaac.2014.08.006

18. van Voorhees EE, Dennis MF, Calhoun PS, Beckham JC. Association of DHEA DHEAS, and cortisol with childhood trauma exposure and post-traumatic stress disorder. Int Clin Psychopharmacol. 2014;29:56-62. doi:10.1097/YIC. Ob013e328364ecd1.

19. Staufenbiel SM, Penninx BW, Spijker AT, Elzinga BM, van Rossum EF. Hair cortisol, stress exposure, and mental health in humans: a systematic review. Psychoneuroendocrinology. 2013;38:1220-35. doi:10.1016/j.psyneuen.2012.11.015.

20. Gao W, Stalder T, Foley P, Rauh M, Deng H, Kirschbaum C. Quantitative analysis of steroid hormones in human hair using a column-switching LC-APCI-MS/ MS assay. J Chromatogr B. 2013;928:1-8. doi:10.1016/j.jchromb.2013.03.008.

21. Stalder T, Kirschbaum C. Analysis of cortisol in hair - state of the art and future directions. Brain Behav Immun. 2012;26:1019-29. doi:10.1016/j.bbi. 2012.02.002

22. Hinkelmann K, Muhtz C, Dettenborn L, Agorastos A, Wingenfeld K, Spitzer C, et al. Association between childhood trauma and low hair cortisol in depressed patients and healthy control subjects. Biol Psychiatry. 2013;74:e15-7. doi:10.1016/j.biopsych.2013.04.021.

23. Kalmakis KA, Meyer JS, Chiodo L, Leung K. Adverse childhood experiences and chronic hypothalamic-pituitary-adrenal activity. Stress. 2015;18:446-50. doi:10.3109/10253890.2015.1023791.

24. Kellner M, Muhtz C, Peter F, Dunker S, Wiedemann K, Yassouridis A. Increased DHEA and DHEA-S plasma levels in patients with post-traumatic stress disorder and a history of childhood abuse. J Psychiatr Res. 2010:44:215-9. doi:10.1016/j.jpsychires.2009.08.009.

25. Bicanic IAE, Postma RM, Sinnema G, Roos C de, Olff M, van Wesel F, van de Putte EM. Salivary cortisol and dehydroepiandrosterone sulfate in adolescent rape victims with post traumatic stress disorder. Psychoneuroendocrinology 2013:38:408-415. doi:10.1016/j.psyneuen.2012.06.015.

26. Usta MB, Tuncel OK, Akbas S, Aydin B, Say GN. Decreased dehydroepiandrosterone sulphate levels in adolescents with post-traumatic stress disorder after single sexual trauma. Nord J Psychiatry. 2016;70:116-20. doi:10.3109/08039488.2015.1056752.

27. Yehuda R, Engel SM, Brand SR, Seckl J, Marcus SM, Berkowitz GS. Transgenerational effects of posttraumatic stress disorder in babies of mothers exposed to the world trade center attacks during pregnancy. The Journal of Clinical Endocrinology \& Metabolism. 2005;90:4115-8. doi:10.1210/jc.2005-0550.

28. Yehuda R, Teicher MH, Seckl JR, Grossman RA, Morris A, Bierer LM. Parental posttraumatic stress disorder as a vulnerability factor for low cortisol trait in offspring of holocaust survivors. Arch Gen Psychiatry. 2007;64:1040. doi:10.1001/archpsyc.64.9.1040

29. Entringer S, Buss C, Wadhwa PD. Prenatal stress, development, health and disease risk: a psychobiological perspective - 2015 Curt Richter award paper. Psychoneuroendocrinology. 2015;62:366-75.

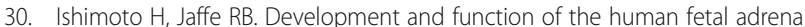
cortex: a key component in the feto-placental unit. Endocr Rev. 2011;32:317-55. doi:10.1210/er.2010-0001.

31. Mesiano S, Jaffe RB. Developmental and functional biology of the primate fetal adrenal cortex. Endocr Rev. 1997;18:378-403. doi:10.1210/edrv.18.3.0304.

32. Rainey WE, Rehman KS, Carr BR. The human fetal adrenal: making adrenal androgens for placental estrogens. Semin Reprod Med. 2004;22:327-36.

33. Mastorakos G, Ilias I. Maternal and fetal hypothalamic-pituitary-adrenal axes during pregnancy and postpartum. Ann N Y Acad Sci. 2003;997:136-49. doi:10.1196/annals.1290.016.

34. Pasqualini JR, Chetrite GS. The formation and transformation of hormones in maternal, placental and fetal compartments: biological implications. Horm Mol Biol Clin Investig. 2016;27:11-28. doi:10.1515/hmbci-2016-0036.

35. Bader K, Hänny C, Schäfer V, Neuckel A, Kuhl C. Childhood Trauma Questionnaire - Psychometrische Eigenschaften einer deutschsprachigen Version. Z Klin Psychol Psychother. 2009:38:223-30. doi:10.1026/16163443.38.4.223. 
36. Teicher MH, Parigger A. The 'Maltreatment and abuse Chronology of Exposure' (MACE) scale for the retrospective assessment of abuse and neglect during development. PLoS One. 2015;10:e0117423. doi:10.1371/journal.pone.0117423.

37. Isele D, Teicher MH, Ruf-Leuschner M, Elbert T, Kolassa I-T, Schury K, et al. KERF-Ein Instrument zur umfassenden Ermittlung belastender Kindheitserfahrungen. Z Klin Psychol Psychother. 2014;43:121-30. doi:10.1026/1616-3443/a000257.

38. Cohen S, Kamarck T, Mermelstein R. A global measure of perceived stress. J Health Soc Behav. 1983:24:385-96.

39. Cooper GAA, Kronstrand R, Kintz P. Society of Hair Testing guidelines for drug testing in hair. Forensic Sci Int. 2012;218:20-4. doi:10.1016/j.forsciint.2011.10.024.

40. Wennig R. Potential problems with the interpretation of hair analysis results. Forensic Sci Int. 2000;107:5-12. doi:10.1016/S0379-0738(99)00146-2.

41. Gareri J, Koren G. Prenatal hair development: implications for drug exposure determination. Forensic Sci Int. 2010;196:27-31. doi:10.1016/j.forsciint.2009. 12.024 .

42. R Core Team. R: A language and environment for statistical computing. Vienna, Austria: R Foundation for; 2015.

43. Rana S, Midi H, Imon AHMR. Robust wild bootstrap for stabilizing the variance of parameter estimates in heteroscedastic regression models in the presence of outliers. Math Probl Eng. 2012;2012:1-14. doi:10.1155/2012/730328.

44. Xiao-Feng Wang. fANCOVA: nonparametric analysis of covariance; 2010.

45. Liu RY. Bootstrap procedures under some non-i.i.d. models. The annals of statistic. 1988;16:1696-708. doi:10.1214/aos/1176351062.

46. Akaike $\mathrm{H}$. A new look at the statistical model identification. IEEE Trans Autom Control. 1974;19:716-23. doi:10.1109/TAC.1974.1100705.

47. Dixon WJ. Analysis of extreme values. Ann Math Statist. 1950;21:488-506.

48. Komsta L. R - package 'outliers'; 2015.

49. Bernstein DP, Stein JA, Newcomb MD, Walker E, Pogge D, Ahluvalia T, et al. Development and validation of a brief screening version of the childhood trauma Questionnaire. Child Abuse Negl. 2003;27:169-90. doi:10.1016/S0145-2134(02)00541-0.

50. Bossé S, Masciotra V, Solomon C, Stalder T, D'Antono B. Childhood trauma, perceived stress, and hair cortisol in adults with and without coronary artery disease. Psychoneuroendocrinology. 2015;61:36. doi:10.1016/j.psyneuen. 2015.07.488

51. Schreier HMC, Bosquet Enlow M, Ritz T, Gennings C, Wright RJ. Childhood abuse is associated with increased hair cortisol levels among urban pregnant women. J Epidemiol Community Health. 2015;69:1169-74. doi:10.1136/jech-2015-205541.

52. Gill J, Vythilingam M, Page GG. Low cortisol, high DHEA, and high levels of stimulated TNF-a, and IL-6 in women with PTSD. J Traum Stress. 2008;21: 530-9. doi:10.1002/jts.20372.

53. Yehuda R, Brand SR, Golier JA, Yang R-K. Clinical correlates of DHEA associated with post-traumatic stress disorder. Acta Psychiatr Scand. 2006;114:187-93. doi:10.1111/j.1600-0447.2006.00801.x.

54. Bremner D, Vermetten E, Kelley ME. Cortisol, dehydroepiandrosterone, and estradiol measured over 24 hours in women with childhood sexual abuserelated posttraumatic stress disorder. J Nerv Ment Dis. 2007;195:919-27. doi:10.1097/NMD.0b013e3181594ca0.

55. Yehuda R, Teicher MH, Seckl J, Grassman RA, Morris A, Bierer LM. Parental posttraumatic stress disorder as a vulnerability factor for low cortisol trait in offspring of holocaust survivors. Arch Gen Psychiatry. 2007;64:1040-8.

56. Tegethoff M, Raul J-S, Jamey C, Khelil MB, Ludes B, Meinlschmidt G. Dehydroepiandrosterone in nails of infants: a potential biomarker of intrauterine responses to maternal stress. Biol Psychol. 2011;87:414-20. doi:10.1016/j.biopsycho.2011.05.007.

57. Kapoor A, Dunn E, Kostaki A, Andrews MH, Matthews SG. Fetal programming of hypothalamo-pituitary-adrenal function: prenatal stress and glucocorticoids. J Physiol. 2006;572:31-44. doi:10.1113/jphysiol.2006.105254.

58. O'Connor TG, Bergman K, Sarkar P, Glover V. Prenatal cortisol exposure predicts infant cortisol response to acute stress. Dev Psychobiol. 2013;55:145-55. doi:10.1002/dev.21007.

59. Zijlmans MAC, Riksen-Walraven JM, de Weerth C. Associations between maternal prenatal cortisol concentrations and child outcomes: a systematic review. Neurosci Biobehav Rev. 2015:53:1-24. doi:10.1016/.jneubiorev.2015.02.015.

60. Wadhwa PD. Psychoneuroendocrine processes in human pregnancy influence fetal development and health. Psychoneuroendocrinology. 2005;30:724-43. doi:10.1016/j.psyneuen.2005.02.004.
61. Wadhwa PD, Entringer S, Buss C, Lu MC. The contribution of maternal stress to preterm birth: issues and considerations. Clin Perinatol. 2011;38:351-84. doi:10.1016/j.clp.2011.06.007.

62. Gluckman PD, Hanson MA, Spencer HG, Bateson P. Environmental influences during development and their later consequences for health and disease: implications for the interpretation of empirical studies. Proc Biol Sci. 2005:272:671-7. doi:10.1098/rspb.2004.3001.

63. Chapman K, Holmes M, Seckl J. 11 beta-hydroxysteroid dehydrogenases: intracellular gate-keepers of tissue glucocorticoid action. Physiol Rev. 2013; 93:1139-206. doi:10.1152/physrev.00020.2012.

64. Ghaemmaghami P, Dainese SM, La Marca R, Zimmermann R, Ehlert U. The association between the acute psychobiological stress response in second trimester pregnant women, amniotic fluid glucocorticoids, and neonatal birth outcome. Dev Psychobiol. 2014;56:734-47. doi:10.1002/dev.21142.

65. Murphy VE, Smith R, Giles WB, Clifton VL. Endocrine regulation of human fetal growth: the role of the mother, placenta, and fetus. Endocr Rev. 2006; 27:141-69. doi:10.1210/er.2005-0011.

66. LeBeau MA, Montgomery MA, Brewer JD. The role of variations in growth rate and sample collection on interpreting results of segmental analyses of hair. Forensic Sci Int. 2011;210:110-6. doi:10.1016/j.forsciint.2011.02.015.

67. Gow R, Thomson S, Rieder M, van Uum S, Koren G. An assessment of cortisol analysis in hair and its clinical applications. Forensic Sci Int. 2010;196:32-7. doi:10.1016/j.forsciint.2009.12.040.

68. Russell E, Kirschbaum C, Laudenslager ML, Stalder T, de Rijke $Y$, van Rossum EF, Elisabeth FC, et al. Toward standardization of hair cortisol measurement; Results of the first international inter-laboratory round robin Ther Drug Monit 2015;37:71-75. doi:10.1097/FTD.0000000000000148.

69. Russell E, Koren G, Rieder M, van Uum S. Hair cortisol as a biological marker of chronic stress: current status, future directions and unanswered questions. Psychoneuroendocrinology. 2012;37:589-601. doi:10.1016/j.psyneuen.2011.09.009.

70. Wippert P-M, Wang HV, Kirschbaum C. Assessment of chronic stress: comparison of hair biomarkers and allostatic load indices. Psychol Res. 2014:4:517-24.

71. Grass J, Kirschbaum C, Miller R, Gao W, Steudte-Schmiedgen S, Stalder T. Sweat-inducing physiological challenges do not result in acute changes in hair cortisol concentrations. Psychoneuroendocrinology. 2015:53:108-16. doi:10.1016/j.psyneuen.2014.12.023.

72. Pragst $F$, Balikova MA. State of the art in hair analysis for detection of drug and alcohol abuse. Clin Chim Acta. 2006;370:17-49. doi:10.1016/j.cca.2006.02.019.

73. Stalder T, Kirschbaum C. Analysis of cortisol in hair - state of the art and future directions. Brain Behav Immun. 2012;26:1019-29. doi:10.1016/j.bbi. 2012.02.002 